\title{
ADEQUAÇÃO DO PRÉ-CONDICIONADOR ILUT PARA SOLUÇÃO DO SUBPROBLEMA LINEAR DO FLUXO DE CARGA VIA GMRES
}

\author{
Carlos Portugal* \\ portugaldele.puc-rio.br \\ Ricardo Prada* \\ pradadele.puc-rio.br \\ José E. O. Pessanha ${ }^{\dagger}$ \\ pessanha@dee.ufma.br \\ *PUC-Rio, DEE, Rua Marquês de São Vicente, 225, Gávea, Rio de Janeiro, RJ - Brasil - 22453-900 \\ †IEE-DEE-UFMA, Avenida dos Portugueses s/n, Campus do Bacanga, São Luís, Ma, - Brasil - 65080-040
}

\section{RESUMO}

Este trabalho propõe uma estratégia de eliminação de elementos não-nulos durante a construção de um pré-condicionador ILU fazendo uso de dois critérios; um baseado no erro gerado para eliminar os elementos do fator $\mathrm{L}$ e o outro numa tolerância relativa para eliminar os elementos de U. Normalmente, esses critérios estão baseados nos níveis dos elementos não-nulos, nos seus valores absolutos, quantidade, ou numa combinação dessas duas últimas. Para o subproblema linear do fluxo de carga esta última combinação pode introduzir elevados erros devido à eliminação inadequada de elementos não-nulos grandes em valor absoluto, aumentando o número de operações efetuadas pelo método iterativo e, conseqüentemente, o tempo de CPU, comprometendo o seu desempenho. É mostrada a influência do esquema de reordenamento neste cenário e experimentos numéricos tipificam o problema e corroboram a melhor qualidade da regra proposta.

PALAVRAS-CHAVE: Pré-condicionadores, Fatoração Incompleta, Métodos Iterativos do Subespaço Krylov, Equações Algébricas Lineares, Fluxo de Carga.

Artigo submetido em 30/12/2009 (Id.: 01095)

Revisado em 27/03/2010, 05/06/2010, 08/06/2010

Aceito sob recomendação do Editor Associado Prof. Takashi Yoneyama

\section{ABSTRACT}

Adequating the ILUT Preconditioner for the Solution of Power Flow Sublinear Problem Using GMRES

This paper proposes a fill-in dropping strategy during ILU preconditioner construction making use of two criteria; one to eliminate the $\mathrm{L}$ factor fill-ins based on the resulting error and the other based on a relative tolerance for $U$ fill-ins. Normally, the fill-in dropping rule is based on levels of fills, numerical values, amount, or a combination of the former two. This combination rule is very well-documented in the literature but for solving the load flow sublinear problem this conventional rule may introduce large errors due to inadequate droppings, increasing the number of operations and the CPU time as well, turning poor the iterative method performance. It is shown the influence of the reordering scheme over that scenario and numerical experiments typify the problem and corroborate the better quality of the proposed rule.

KEYWORDS: Preconditioners, Incomplete Factorization, Krylov subspace iterative methods, Algebraic Linear Equations, Load-Flow.

\section{INTRODUÇÃO}

A aplicação de métodos iterativos do subespaço Krylov pré-condicionados tem sido direcionada basicamente para solucionar sistemas lineares do tipo A. $\mathrm{x}=\mathrm{b}$ complexos e com milhões de incógnitas (Axelsson, 1994; Barrett, 2006; 
Benzi, 2002; Saad, 2003; Van Der Vorst, 2003). Um método iterativo se adequadamente pré-condicionado pode ser aplicado eficientemente em sistemas de menor porte (milhares de incógnitas) e com matrizes mal-condicionadas. Entretanto, encontrar um pré-condicionador para solucionar problemas com estas características não é uma tarefa simples. Uma das principais dificuldades está no fato do pré-condicionamento se basear em aproximações e, na ausência de informações precisas do comportamento da solução e das propriedades da matriz de coeficientes, a convergência pode ficar muito dependente de informações perdidas ao longo do processo de aproximação. A qualidade do pré-condicionador pode piorar em função de qualquer mudança no comportamento da solução, reduzindo a taxa de convergência e a robustez do método iterativo, aumentando o número de operações efetuadas ao longo do processo de solução. O método também pode falhar e não solucionar o sistema linear considerando-se os parâmetros estabelecidos, como tolerância e número máximo de iterações. Devido à importância dos pré-condicionadores para os métodos iterativos, estes se tornaram uma interessante e desafiadora linha de pesquisa, estando sob constante investigação (Benzi, Haws, \& Tuma, 2000; Benzi, 2002; Bruaset, 1995; Chen, 2005; Greenbaum, 1997).

A elaboração de um pré-condicionador pode ser feita basicamente por dois caminhos; um estabelecendo forte dependência com as características do problema e o outro com menos. A vantagem da primeira opção está na boa qualidade do pré-condicionador. Por outro lado, como já informado, qualquer mudança nas características do problema pode afetar negativamente a sua qualidade comprometendo o desempenho do método iterativo. A segunda opção abrange um pré-condicionador menos dependente, podendo ser aplicado a diferentes tipos de problemas. Entretanto, apesar do seu processo de construção ser menos complexo, a sua qualidade pode ser comprometida devido à problemas de precisão e instabilidade numérica, principalmente se baseado em fatores triangulares inferior (L) e superior (U) incompletos. Pré-condicionadores que fazem uso desta forma de construção são conhecidos como ILU (Saad, 1996) e diferem no critério para eliminar elementos não-nulos que surgem durante o processo de fatoração em posições antes ocupadas por elementos nulos. O presente trabalho investiga um desses critérios (ou regras) quando considerado na solução iterativa do subproblema linear do fluxo de carga via método GMRES (Saad \& Schultz, 1986) com o pré-condicionador construído a partir da matriz Jacobiana calculada na primeira iteração do método de Newton-Raphson. A matriz Jacobiana é formada por quatro submatrizes identificadas como $H, N$, $M$ e $L$ em (1), sendo estas estruturalmente idênticas e simétricas; conseqüentemente, a matriz Jacobiana também é estruturalmente simétrica, embora seja numericamente assimétrica (Prada, 1977; Monticelli, 1983; Borges \& Coutinho, 1996). Outra característica relevante da matriz Jacobiana é sua esparsidade. Esta característica é inerente às redes elétricas dado que cada barra pode ter apenas alguns poucos ramos conectados a ela. Visto que o número médio de ramos conectados às barras é o mesmo, independentemente do tamanho do sistema, então, quanto maior for o sistema, maior será o número de elementos nulos na matriz Jacobiana, tornando-se cada vez mais esparsa. Em (1) $\Delta P$ e $\Delta Q$ correspondem aos erros das potências ativa e reativa, $V \mathrm{e}$ $\theta$ ao módulo e ângulo da tensão da barra, respectivamente.

$$
J=-\left[\begin{array}{ll}
\frac{\partial \Delta P}{\partial \theta} & \frac{\partial \Delta P}{\partial V} \\
\frac{\partial \Delta Q}{\partial \theta} & \frac{\partial \Delta Q}{\partial V}
\end{array}\right]=-\left[\begin{array}{cc}
H & N \\
M & L
\end{array}\right]
$$

A regra de eliminação de elementos não-nulos investigada e identificada neste trabalho como "convencional" foi proposta por (Saad, 1994) e o pré-condicionador associado a esta regra é conhecido como $\operatorname{ILUT}(\tau, \rho)$. São identificadas deficiências desta regra para o problema de interesse e é proposta uma nova estratégia de eliminação baseada em dois critérios: um, para eliminar os elementos do fator $\mathrm{L}$ com base no erro gerado; o outro, uma variante da regra convencional para eliminar os elementos do fator U. Esta estratégia é identificada neste trabalho como regra composta. Para controlar o número de elementos não-nulos é considerada também uma estratégia de reordenamento. Experimentos numéricos corroboram a melhor eficiência da estratégia de eliminação proposta em relação a convencional em termos de quantidade de elementos eliminados, número de operações de ponto flutuante e tempo de CPU.

\section{O PRÉ-CONDICIONADOR ILUT}

A regra aplicada no pré-condicionador $\operatorname{ILUT}(\tau, \rho)$ considera uma tolerância absoluta $\tau$ e um número $\rho$ de elementos não-nulos permitidos nas linhas dos fatores incompletos. Seu mecanismo se resume a eliminar um elemento (substituído por zero) se este for menor que uma tolerância relativa $\tau_{i}$ obtida multiplicando-se $\tau$ pela norma-2 da i-ésima linha. São mantidos apenas os $\rho$ maiores elementos no fator $\mathrm{L}$ da linha e os $\rho$ maiores elementos no fator $\mathrm{U}$ da linha em adição ao elemento diagonal, que é sempre mantido. A razão para usar uma tolerância relativa se deve à matrizes mal escalonadas (Benzi, 2002). Apesar de bastante referenciada na literatura (Benzi, 2002; Chen, 2005; Saad, 1994; 1996; 2003; Van Der Vorst, 2003; Zhang; 2000), esta regra pode introduzir erros reduzindo a eficiência do pré-condicionador. As próximas subseções ilustram estes problemas e propõem uma estratégia de eliminação baseada em dois critérios. 


\subsection{Eliminação convencional}

Para ilustrar as deficiências da regra de eliminação convencional para o problema de interesse, simula-se um fluxo de carga utilizando um sistema-teste de pequeno porte de cinco barras e dois geradores (CEPEL, 2001). Inicialmente, dois experimentos numéricos foram realizados para diferentes tolerâncias absolutas, $\tau_{1}=2 \times 10^{-4} \mathrm{e}$ $\tau_{2}=2,5 \times 10^{-4}$, e como o sistema é de pequeno porte o parâmetro $\rho$ não foi considerado, não havendo, portanto, limite máximo para o número de elementos não-nulos permitidos. A Tabela 1 mostra os elementos do fator $\mathrm{L}$ (fator triangular inferior) em ordem crescente baseada em seus valores absolutos (colunas um e dois), quantos e quais foram eliminados (marcados por um X) em função das tolerâncias (coluna três) e o erro gerado por cada eliminação (coluna quatro), este computado de acordo com (2), onde $\bar{L}$ representa o fator triangular inferior incompleto (Benzi, Szyld \& Van Duin, 1999; Benzi, 2002).

$$
\operatorname{Erro}\left(l_{i k}\right)=\|L \cdot U-\bar{L} \cdot U\|_{1}, i>k
$$

Tabela 1: Eliminação - regra convencional

\begin{tabular}{|c|c|c|c|c|}
\hline$l_{i j}$ & $\left|l_{i j}\right|$ & \multicolumn{2}{|c|}{ Tolerâncias } & Erro \\
\hline- & - & $\tau_{1}$ & $\tau_{2}$ & - \\
\hline$l_{10,2}$ & $1,31 \times 10^{-18}$ & $\mathrm{X}$ & $\mathrm{X}$ & 0,0010 \\
\hline$l_{6,5}$ & $7,62 \times 10^{-16}$ & $\mathrm{X}$ & $\mathrm{X}$ & 0,0018 \\
\hline$l_{8,6}$ & $7,66 \times 10^{-16}$ & $\mathrm{X}$ & $\mathrm{X}$ & 0,0018 \\
\hline$l_{9,5}$ & $7,66 \times 10^{-16}$ & $\mathrm{X}$ & $\mathrm{X}$ & 0,0018 \\
\hline$l_{8,5}$ & $6,62 \times 10^{-15}$ & $\mathrm{X}$ & $\mathrm{X}$ & 0,0076 \\
\hline$l_{9,6}$ & $6,62 \times 10^{-15}$ & $\mathrm{X}$ & $\mathrm{X}$ & 0,0076 \\
\hline$l_{7,2}$ & $6,68 \times 10^{-15}$ & $\mathrm{X}$ & $\mathrm{X}$ & 0,0077 \\
\hline$l_{10,4}$ & 0,0095 & - & - & 0,0328 \\
\hline$l_{9,8}$ & 0,0156 & - & - & 3,2377 \\
\hline$l_{8,7}$ & 0,0241 & - & - & 4,8525 \\
\hline$l_{10,7}$ & 0,0247 & - & - & 4,9734 \\
\hline$l_{7,3}$ & 0,0328 & - & $\mathrm{X}$ & 8,0816 \\
\hline$l_{4,3}$ & 0,0332 & - & - & 8,1728 \\
\hline$l_{2,1}$ & 0,0612 & - & $\mathrm{X}$ & 4,0129 \\
\hline
\end{tabular}

Os elementos acima da linha em negrito são menores em magnitude que as tolerâncias absolutas e os abaixo são maiores. Para o $\operatorname{ILUT}\left(\tau_{1}\right)$ foram eliminados os sete menores elementos de L e para o $\operatorname{ILUT}\left(\tau_{2}\right)$, além destes, também $l_{7,3}$ e $l_{2,1}$.

Apesar do problema ter sido resolvido, as conseqüências dessas eliminações acima da tolerância absoluta podem ser melhor visualizadas se computadas as operações de ponto flutuante. Essas informações estão na Tabela 2, onde também estão incluídos os resultados associados ao GMRES não pré-condicionado (NP) e a um pré-condicionador de fatores completos (LU). Sem pré-condicionamento o GMRES é muito ineficiente apresentando um elevado número de operações (16.164). Com um pré-condicionador de fatores completos, o número de operações foi de 413, sendo 199 do pré-condicionador e 214 do GMRES. Com o $\operatorname{ILUT}\left(\tau_{1}\right)$, o número de operações efetuadas para a construção do pré-condicionador diminuiu e as efetuadas pelo GMRES se manteve, totalizando 369. Para o $\operatorname{ILUT}\left(\tau_{2}\right)$, o número de operações efetuadas para a construção do pré-condicionador diminuiu ainda mais e as efetuadas pelo GMRES aumentou, totalizando 571 operações. O aumento da tolerância absoluta para eliminar mais elementos não-nulos reduz o número de operações efetuadas para a construção do pré-condicionador. Por outro lado, a sua qualidade é comprometida, piorando a taxa de convergência do GMRES e aumentando em maiores proporções as operações efetuadas no processo iterativo. Isto ocorreu devido ao fato do mecanismo de eliminação de elementos não-nulos estar baseado numa tolerância relativa (explicado anteriormente), e não na absoluta. Para a tolerância $\tau_{2}$ este mecanismo, a priori, não seguiu a sequiência desejada de eliminação, já que os próximos elementos a serem eliminados deveriam ser $l_{10,4}, l_{9,8}, \ldots$, $l_{2,1}$. Ao invés disso, foram eliminados elementos $l_{7,3}$ e $l_{2,1}$ gerando erros que afetaram negativamente a qualidade do pré-condicionador ILU, justificando o aumento no número de operações efetuadas pelo GMRES.

Tabela 2: Número de operações de ponto flutuante

\begin{tabular}{|c|c|c|c|c|}
\hline & \multirow{2}{*}{ NP } & LU & \multicolumn{2}{|c|}{ ILUT } \\
\cline { 3 - 5 } & & - & $\tau_{1}$ & $\tau_{2}$ \\
\hline Pré-condicionador & - & 199 & 155 & 143 \\
\hline GMRES & 16.164 & 214 & 214 & 428 \\
\hline Total & 16.164 & 413 & 369 & 571 \\
\hline
\end{tabular}

Outra situação interessante está associada ao grande valor do erro resultante da eliminação do elemento $l_{10,2}$. Isto ocorreu em função do modelo de fluxo de carga considerado (Monticelli, 1983) utilizar valores muito elevados na diagonal da matriz para barras de referência e de tensão controlada. Esses valores produzem elementos muito pequenos em $\mathrm{L}$ e grandes em $\mathrm{U}$. Os solucionadores, tanto os diretos como os iterativos, devem ser capazes de contornar esta situação. Sem considerar esses valores elevados, a matriz Jacobiana é apenas mal condicionada. Por outro lado, se forem considerados, a matriz se torna extremamente mal condicionada.

\subsection{Regra de Eliminação Composta}

É proposta uma estratégia de eliminação de elementos não-nulos do fator L baseada no erro gerado a fim de evitar 
os problemas mostrados. Sendo L o fator triangular inferior completo e $\bar{L}$ o incompleto, após a eliminação de um único elemento e reescrevendo (2) como em (3) (Benzi, Szyld \& Van Duin, 1999; Benzi, 2002), verifica-se que $(L-\bar{L})$ possui apenas um elemento não nulo, $\bar{l}_{i k}$ (eliminado em $\bar{L}$ ), como apresentado em (4).

$$
\begin{gathered}
\operatorname{Erro}\left(L_{i k}\right)=\|(L-\bar{L}) \cdot U\|_{1}, i>k \\
(L-\bar{L})=\left[\begin{array}{ccccc}
0 & & & & \\
\vdots & \ddots & & & \\
0 & \cdots & l_{i k} & & \\
\vdots & & \vdots & \ddots & \\
0 & \cdots & 0 & \cdots & 0
\end{array}\right], i>k
\end{gathered}
$$

Substituindo (4) em (3):

$$
(L-\bar{L}) \cdot U=\left[\begin{array}{cccc}
0 & \cdots & 0 & \cdots \\
\vdots & & \vdots & \\
0 & \cdots & l_{i k} \cdot u_{k k} & \cdots \\
\vdots & & \vdots & \\
0 & \cdots & 0 & \cdots \\
& 0 & \cdots & 0 \\
& \vdots & & \vdots \\
& l_{i k} \cdot u_{k i} & \cdots & l_{i k} \cdot u_{k n} \\
& \vdots & & \vdots \\
& 0 & \cdots & 0
\end{array}\right], i>k
$$

Resultando para o cálculo do erro:

$$
\operatorname{Erro}\left(l_{i k}\right)=\left|l_{i k}\right| \cdot \max \left\{u_{k m}\right\}, i>k, m=[k, \ldots, n]
$$

O processo de fatoração usado é conhecido como IKJ e deriva da eliminação Gaussiana (Saad, 2003). Para computar o erro gerado pela eliminação do elemento $l_{i k}$ é necessário conhecer todos os elementos $u_{k m}$, já calculados nos passos anteriores. Já para o erro gerado pelo elemento $u_{i k}$ é necessário conhecer os elementos $l_{m k}$ mas nem todos são conhecidos. Portanto, a regra baseada no erro é aplicada apenas nos elementos do fator $\mathrm{L}$, enquanto que uma variante da regra convencional é usada para os elementos de U. O resultado é um pré-condicionador que aplica dois critérios de eliminação da seguinte forma:
- De acordo com a relação dada por (7), um elemento $l_{i k}$ é eliminado se o erro resultante for menor que uma tolerância $\xi$ usada para todas as linhas de L. Caso contrário, calcula-se $l_{i k}$ usando-se o elemento $a_{i k}^{\prime}$ da matriz resultante do processo de eliminação Gaussiana até a correspondente etapa. Já para um elemento $u_{i k}$, este é eliminado se for menor que uma tolerância relativa $\tau_{i}$, obtida multiplicando-se uma tolerância absoluta pela média aritmética dos elementos da i-ésima linha da matriz de coeficientes original (8). $O$ cálculo de $\omega$ é efetuado de acordo com a fatoração IKJ e seu algoritmo é apresentado no Apêndice A (Saad, 2003).

$$
\underset{k=(1, \ldots, i-1)}{l_{i k}}=\left\{\begin{array}{cc}
0, & \operatorname{Erro}\left(l_{i k}\right)<\xi \\
\frac{a_{i k}^{\prime}}{u_{k k}}, & \operatorname{Erro}\left(l_{i k}\right) \geq \xi
\end{array}\right.
$$

$$
\underset{k=(i+1, \ldots, n)}{u_{i k}}=\left\{\begin{array}{cc}
0, & \omega_{k}<\tau_{i} \\
\omega_{k}, & \omega_{k} \geq \tau_{i}
\end{array}\right.
$$

O cálculo da norma-2 usada na regra convencional de eliminação, que é dada pela raiz quadrada da soma dos quadrados dos elementos da linha, pode exigir considerável esforço computacional dependendo da dimensão da matriz de coeficientes. Portanto, ao invés de se usar esta norma para eliminação dos elementos em $\mathrm{U}$, optou-se pela média aritmética dos elementos da i-ésima linha da matriz de coeficientes original. Este procedimento reduz o número de operações de ponto flutuante, sem comprometer a qualidade do pré-condicionador. No entanto, a eficiência de ambas heurísticas é inferior a da regra baseada no erro proposta aqui para eliminação dos elementos em $\mathrm{L}$.

O método aplicado na fatoração IKJ impede que a eliminação baseada no erro seja também aplicada no fator U. Por outro lado, se L apresentar um maior número de elementos a eliminar, a aplicação da regra baseada no erro apenas em L ainda pode ser vantajoso. Através de três diferentes configurações de sistemas elétricos procurou-se estabelecer uma relação percentual entre os elementos de $\mathrm{L}$ e de $\mathrm{U}$ que estariam abaixo de uma tolerância absoluta $\tau\left(\tau_{1}\right.$ e $\tau_{2}$ estabelecidos anteriormente) e que seriam eliminados. Os resultados estão ilustrados na Figura 1 e confirmam que a maioria dos elementos a eliminar está no fator $\mathrm{L} \mathrm{e}$ que, portanto, a regra proposta pode melhorar a eficiência numérica e computacional do processo iterativo devido a uma melhor qualidade do pré-condicionador ILUT.

O GMRES pode convergir no menor número possível de iterações e operações e neste caso, a não eliminação de elementos não nulos em U não afetaria sua taxa de 
convergência. Porém, caso esses elementos não sejam eliminados, o número de operações de ponto flutuante aumenta e o esforço para a construção do pré-condicionador também.
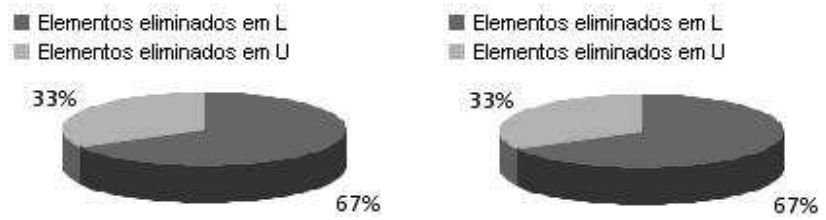

(a) Sistema IEEE-118 barras $\left(\tau_{1}\right.$ e $\left.\tau_{2}\right)$
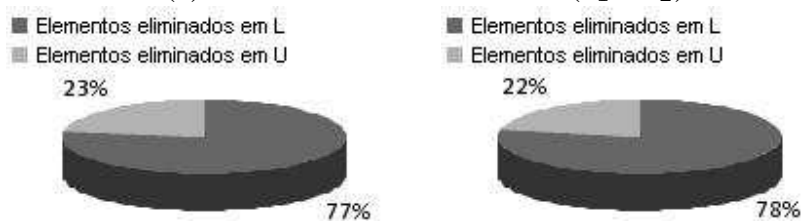

(b) Sistema Norte-Nordeste - 257 barras $\left(\tau_{1}\right.$ e $\left.\tau_{2}\right)$
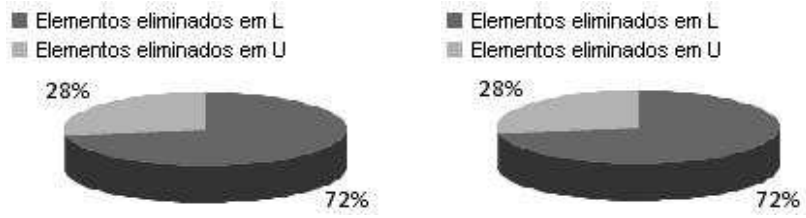

(c) Sistema Brasileiro -3.315 barras $\left(\tau_{1}\right.$ e $\left.\tau_{2}\right)$

Figura 1: Quantidade percentual de eliminação em $L$ e U.

\subsection{Reordenamento}

Pré-condicionadores ILU são sensíveis ao reordenamento da matriz de coeficientes, reduzindo o número de elementos não-nulos (da mesma forma como usado com os métodos diretos) e melhorando a estabilidade numérica da fatoração. Na maioria dos casos, o reordenamento melhora a taxa de convergência dos métodos do subespaço Krylov (Benzi \& Tuma, 2000). As duas classes de métodos mais populares para esta aplicação são o Cuthill-McKee reverso - RCM (Cuthill, 1972) e o Minimum Degree. A primeira confina os elementos não-nulos em uma banda estreita e a segunda minimiza a quantidade de elementos não-nulos analisando o padrão de esparsidade da matriz usando a teoria dos grafos (Watkins, 2002).

Para o problema de fluxo de carga, foi proposto em (Alves et al, 1999) um esquema de reordenamento envolvendo matrizes simétricas definidas positivas que, segundo os autores, é tão eficiente para os métodos iterativos quanto o método de Tinney II (Tinney,1967) que é exemplo dos métodos Minimum Degree, é para os diretos. O RCM foi considerado em (Pessanha, Portugal \& Saavedra,
2010; Flueck \& Chiang, 1998) na construção de um pré-condicionador ILU também para solução do fluxo de carga. No presente trabalho, o desempenho desses dois métodos é comparado a fim de obter informações mais detalhadas que as apresentadas nessas referências com relação ao reordenamento e aos métodos iterativos. As investigações (experimentos numéricos) da próxima seção estão baseadas na taxa de convergência do GMRES dada por (9) (Saad, 2003), em função da norma do resíduo a cada iteração linear $i$ dada por (10) onde $\mathrm{r}_{1}$ e $\mathbf{r}_{N i}$ são os resíduos da primeira e da última iteração linear convergida e Ni é o número total de iterações lineares. Ainda em (10) , A é uma matriz de coeficientes $n \times n$ e b um vetor de dimensão- $n$. Solucionando o subproblema linear do fluxo de carga pré-condicionando o sistema original pela esquerda através da matriz de transformação $M$, e incluindo reordenamento através da matriz $\mathrm{P}$, o resíduo passa a ser expresso por (11), onde $\mathrm{J}$ é a matriz Jacobiana na k-ésima iteração Newton-Raphson e $\mathrm{x}_{i}$ a solução procurada (Pessanha, Portugal \& Saavedra, 2010).

$$
T C=\frac{\log _{10}\left\|r_{1}\right\| /\left\|r_{N i}\right\|}{N i-1}
$$

$$
\left\|r_{i}\right\|_{2}=\left\|b_{k}-A_{k} \cdot x_{i}\right\|
$$

$$
r_{i}=\underbrace{M_{k}^{-1} \cdot P^{T} \cdot\left[\begin{array}{c}
\Delta P \\
\Delta Q
\end{array}\right]^{k}}_{b_{k}}-\underbrace{M_{k}^{-1} \cdot\left[P^{T} \cdot J_{k} \cdot P\right]}_{A_{k}} \cdot \underbrace{\left[\Delta Z_{i}\right]}_{x_{i}}
$$

\section{EXPERIMENTOS NUMÉRICOS}

É avaliado o desempenho do método iterativo GMRES pré-condicionado na solução do subproblema linear do fluxo de carga considerando a estratégia de eliminação composta. É usada uma configuração do sistema elétrico brasileiro com 3.515 barras e 301 geradores no cenário de carga pesada, resultando numa matriz Jacobiana da ordem de $7.030 \times$ 7.030 .

Nos experimentos numéricos são analisados quatro indicadores: a taxa de convergência, o número em milhões de operações de ponto flutuante (MFlops), o tempo de CPU e o número de novos elementos não-nulos. Em todos os experimentos foram usadas como tolerâncias os valores $10^{-3}$ e $10^{-5}$ para os sistemas não-lineares e lineares (absoluta e relativa), respectivamente. Aqui, vale notar que, 
a tolerância dos sistemas não-lineares influencia no número de sistemas lineares a ser resolvido pelo GMRES. Quanto menor for esta tolerância, maior será o número de iterações do método Newton-Raphson para atingir a precisão desejada e consequientemente maior será o número de sistemas lineares a ser resolvido. O número máximo de iterações foi de 15 para sistemas não-lineares e de 100 para os lineares (GMRES).

\subsection{Parâmetros do Pré-Condicionador}

É necessário cautela com relação aos valores dos parâmetros das regras de eliminação (convencional e composta) para se construir o pré-condicionador. Este é um aspecto crítico quando se usa pré-condicionadores baseados em fatoração incompleta, pois valores não adequados podem eliminar mais, ou menos, elementos não nulos do que o necessário, refletindo negativamente na qualidade do pré-condicionador e conseqüentemente na eficiência e robustez do método iterativo. Para ilustrar os efeitos dos parâmetros das regras desde a construção do pré-condicionador até a convergência do GMRES, foram simulados 75.076 fluxos de carga considerando diferentes $\rho$ e $\tau$, e 112.225 fluxos para diferentes $\xi$ e $\tau$. Os resultados estão ilustrados na Figura 2 sob forma de gráficos cujas tonalidades estão associadas ao número de operações efetuadas. A região mais escura em ambos os gráficos corresponde às simulações que resultaram no menor número de operações de ponto flutuante. Portanto, qualquer par desta região a priori, pode ser considerado para futuras simulações (como assumido para os Casos subseqüentes). Já as regiões mais claras correspondem às simulações que resultaram nos maiores números de operações de ponto flutuante. As barras laterais aos gráficos fornecem informações normalizadas sobre a evolução do número de operações à medida que os parâmetros se afastam das regiões mais escuras até a branca, ou seja, a de não convergência (NC). São informados também, os números mínimo e máximo de operações em MegaFlops (MFlops) para cada estratégia de eliminação onde se nota que a composta efetua menos operações em qualquer extremidade (mínimo e máximo).

Como já informado, os parâmetros (Apêndice B) considerados nos próximos experimentos numéricos fazem parte da região mais escura, isto é, a de menor número de operações. Portanto, usou-se o quê de melhor cada regra poderia oferecer, tornando de certa forma mais justas as comparações entre as regras (Caso II).

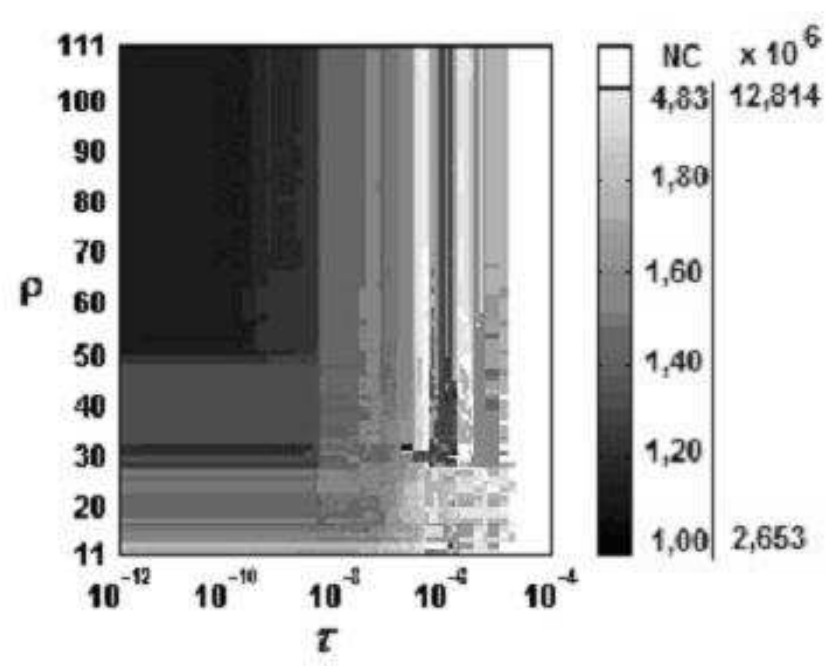

(a) Parâmetros $\rho$ e $\tau$

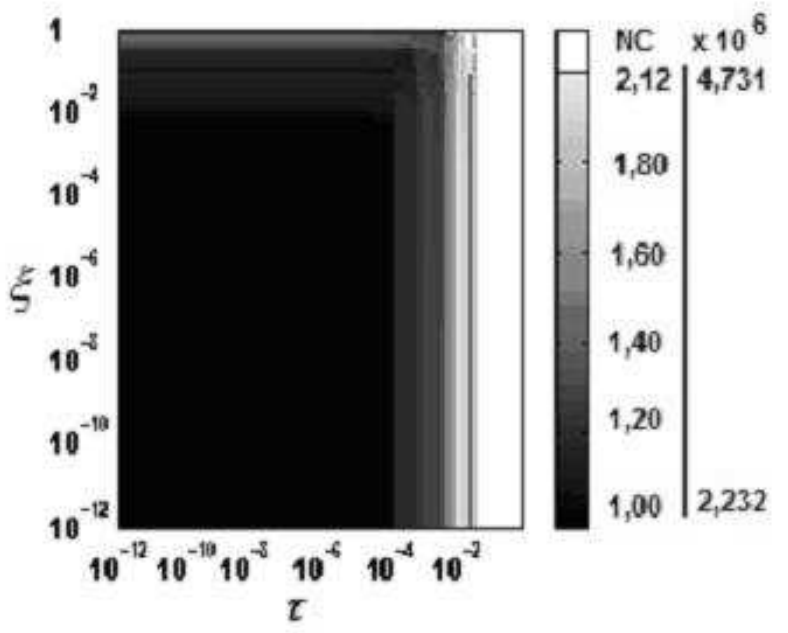

(a) Parâmetros $\xi$ e $\tau$

Figura 2: Indicadores de operações de ponto flutuante

\subsection{Caso I: Estratégias de Reordenamento}

Este caso investiga a influência das estratégias de reordenamento Tinney II, que faz parte da classe de métodos minimum degree, e o RCM, quando aplicadas na matriz Jacobiana calculada e usada na construção do pré-condicionador. A Tabela 3 informa o número de elementos não-nulos incluídos nos fatores triangulares do pré-condicionador para cada tipo de reordenamento. Comparado ao RCM, o método de Tinney II (Minimum Degree) evitou 36.645 elementos não-nulos no pré-condicionador. A redução do número desses elementos após o reordenamento diminui o esforço computacional associado à construção do pré-condicionador, como 
informado na Figura 3 pelo número de operações efetuadas (MFlops). A estratégia de reordenamento Tinney II resulta num menor número de operações e menor tempo de CPU, apesar da construção do pré-condicionador exigir maior esforço que o gasto pelo GMRES. Sem reordenamento o tempo de CPU foi de $25,43 \mathrm{~s}$.

Os gráficos da Figura 4 oferecem informações percentuais sobre os elementos não-nulos eliminados e os não eliminados pela regra convencional associada ao pré-condicionador ILUT para cada estratégia de reordenamento. Usando-se o método de Tinney II, a quantidade de elementos não-nulos eliminados foi de apenas 2,95\%, menos eliminações em termos percentuais se comparado ao RCM. Entretanto, o número de elementos não-nulos resultantes do reordenamento Tinney II é bem menor que do RCM (ver informações da Tabela 3). A influência do reordenamento na taxa de convergência do GMRES é mostrada através do gráfico da Figura 5, confirmando não apenas o seu efeito positivo, mas também a superioridade do método de Tinney II.

Tabela 3: Elementos não-nulos: Caso I

\begin{tabular}{|c|c|c|}
\hline Sem Reordenamento & RCM & Tinney II \\
\hline 3.435 .437 & 52.083 & 15.438 \\
\hline
\end{tabular}

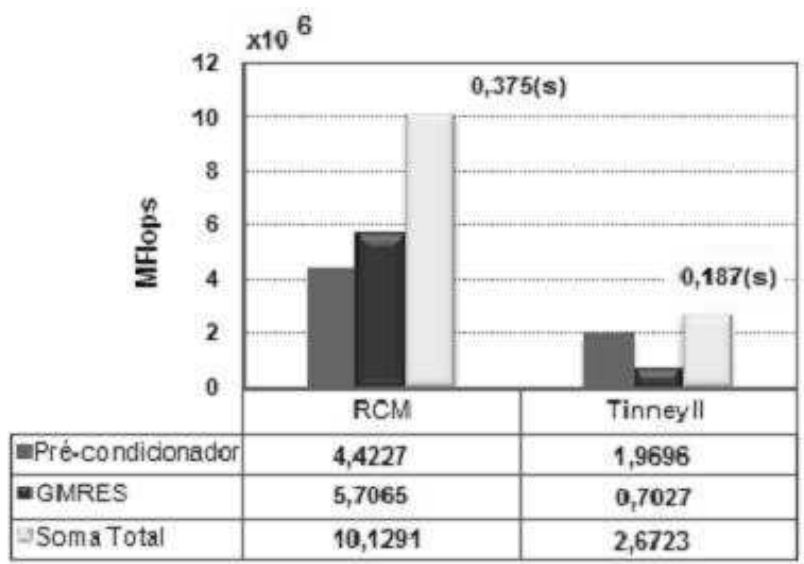

Figura 3: Número de operações e tempo de CPU: Caso I

\subsection{Caso II: Regra Composta}

Apesar do reordenamento Tinney II evitar um grande número de elementos não-nulos nos fatores triangulares do pré-condicionador ILU, este último ainda deve fazer uso de uma regra de eliminação para manter a característica dos fatores triangulares incompletos. Ainda, fazendo uso apenas do reordenamento (sem pré-condicionamento), a

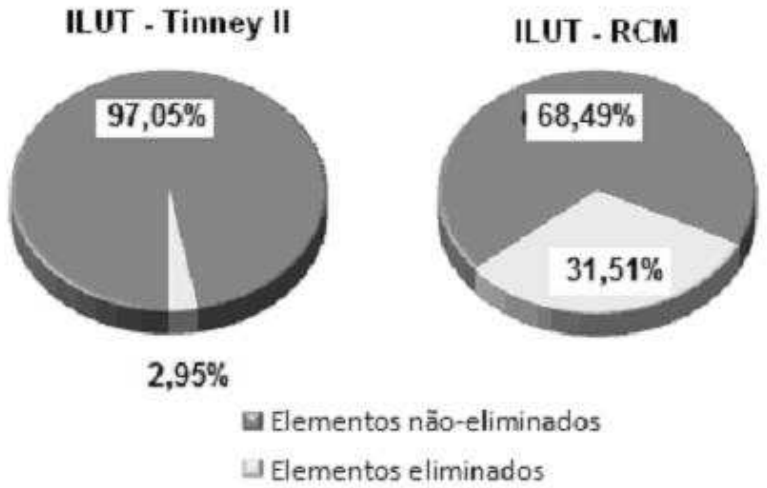

Figura 4: Elementos eliminados: Caso I

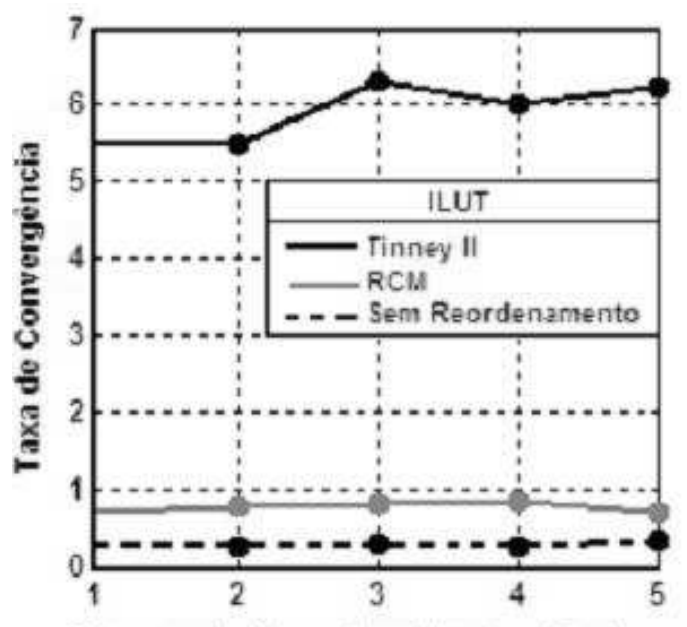

Número de iteraçōes Newton-Raphson

Figura 5: Taxa de convergência do GMRES - regra de eliminação convencional

eficiência computacional do processo iterativo do GMRES pode ser bastante prejudicada, ou até mesmo não solucionar o problema. Ambas as situações foram observadas ao longo das investigações.

No presente conjunto de simulações é testada a estratégia de eliminação proposta e o resultado é comparado com a convencional. Disponibiliza-se também o resultado com o pré-condicionador LU (sem eliminação). A estratégia de reordenamento considerada é Tinney II por ter apresentado melhores resultados (Caso I). A Tabela 4 informa a quantidade de elementos não-nulos e a Figura 6 o número de operações de ponto flutuante e o tempo de CPU para solucionar o subproblema linear. A eliminação baseada na estratégia composta apresenta o menor número de elementos não-nulos, menor número de operações e menor tempo de CPU mostrando, neste caso, ser mais eficiente que a regra 
convencional.

Os gráficos da Figura 7 oferecem informações percentuais sobre o processo de eliminação, onde se nota um aumento na quantidade de elementos eliminados pela regra composta em comparação com a regra convencional. Portanto, se a regra composta for adequadamente combinada com o reordenamento Tinney II, mais elementos são eliminados sem comprometer a qualidade da solução e melhorando a taxa de convergência do GMRES, reduzindo o número de operações efetuadas durante o processo iterativo.

Finalmente, a Tabela 5 informa o ganho percentual para cada estratégia em termos de eficiência computacional com base no maior tempo de CPU (sem eliminação e com pré-condicionador LU).

Tabela 4: Elementos não-nulos: Caso II

\begin{tabular}{|c|c|c|}
\hline $\begin{array}{c}\text { Eliminação } \\
\text { Convencional }\end{array}$ & $\begin{array}{c}\text { Eliminação } \\
\text { Composta }\end{array}$ & Sem Eliminação \\
\hline 15.438 & 14.720 & 15.794 \\
\hline
\end{tabular}

Tabela 5: Eficiência computacional (Ganho)

\begin{tabular}{|c|c|}
\hline Eliminação Convencional & Eliminação Composta \\
\hline $3,1 \%$ & $32,6 \%$ \\
\hline
\end{tabular}

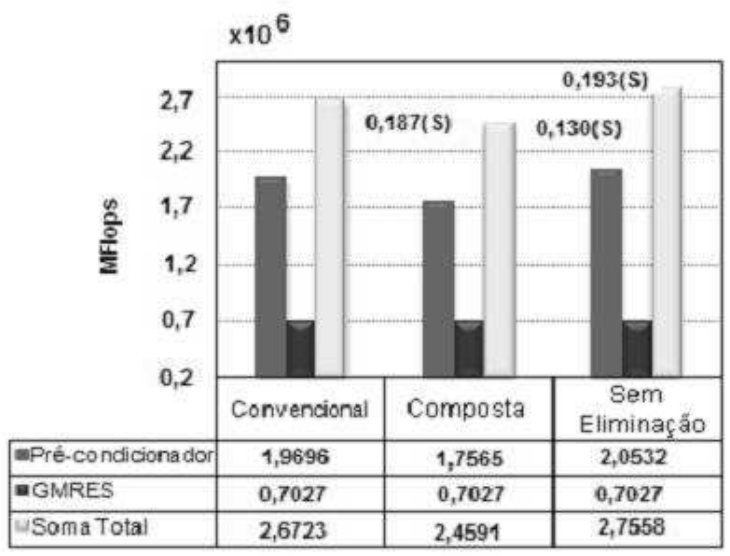

Figura 6: Número de operações e tempo de CPU: Caso II

\section{CONCLUSÕES}

Visando um processo iterativo (GMRES) pré-condicionado eficiente para solucionar o subproblema linear do fluxo de carga, o presente artigo propôs uma estratégia para eliminar elementos não-nulos durante a construção de um
Regra Convencional

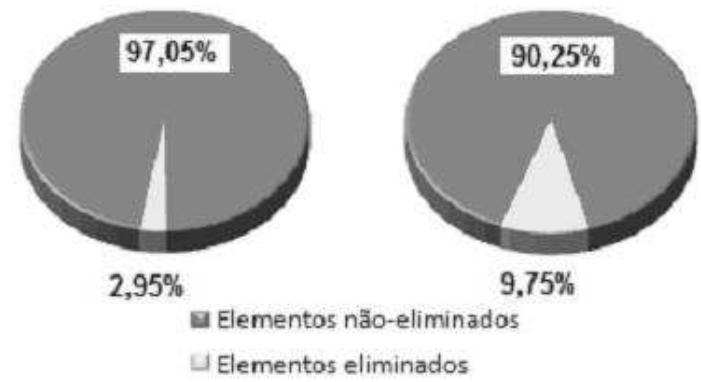

Figura 7: Elementos eliminados: Caso II. pré-condicionador ILUT fazendo uso de dois critérios; um baseado no erro gerado para eliminar os elementos do fator L e o outro numa tolerância relativa obtida pelo produto da tolerância absoluta pela média aritmética dos elementos da i-ésima linha da matriz de coeficientes para eliminar os elementos de U. A necessidade de se apresentar tal proposta está no fato da regra convencional, bastante divulgada na literatura, estar sujeita a gerar grandes erros devido a eliminação inadequada de certos elementos prejudicando a eficiência do processo iterativo, mesmo quando aplicada na solução de sistemas lineares de pequeno-porte.

Foi mostrado também que, com o reordenamento Tinney II o número de elementos não nulos é bem menor em comparação ao obtido com reordenamento RCM, reduzindo o número de operações efetuadas na construção do pré-condicionador e na aplicação do GMRES. Portanto, o reordenamento Tinney II usado na construção do pré-condicionador ILUT junto com a regra de eliminação proposta melhoram o processo de solução devido a um aumento na robustez e na eficiência do método GMRES, resultado de um pré-condicionador de melhor qualidade.

Apesar do problema investigado estar associado a engenharia de sistemas elétricos, acredita-se que a proposta possa ser aplicada em outros problemas científicos, já que o ponto chave está na eliminação de elementos não-nulos do pré-condicionador ILU.

\section{REFERÊNCIAS BIBLIOGRÁFICAS}

Alves, A.B. , Asada, E.N. \& Monticelli, A. (1999). Critical Evaluation of Direct and Iterative Methods for solving $A x=b$ systems in Power Flow Calculations and Contingency Analysis. IEEE Transactions on Power Systems, Vol.14, Ňr 2.

Axelsson, O. (1994). Iterative Solution Methods. 
Cambridge: Cambridge Univ. Press.

Barrett, R.,. (2006). Templates for the Solution of Linear Systems: Building Blocks for Iterative Methods. Philadelphia, PA: SIAM.

Benzi, M., Szyld, D. B., \& Van Duin, A. (1999). Orderings for incomplete factorization preconditioning of nonsymmetric problems. SIAM Journal on Scientific Computing, Vol. 20, Issue 5, pp: $1652-1670$.

Benzi, M., \& Tuma, M. (2000). Orderings for factorized approximate inverse preconditioners. SIAM Journal on Scientific Computing,Vol. 21, Issue 5, pp. 1851 - 1868.

Benzi, M. B., Haws, J. C., \& Tuma, M. (2000). Preconditioning highly indefinite and nonsymmetric matrices. SIAM, J. Sci. Comput. 22, pp. 1333-1353.

Benzi, M. (2002), Preconditioning Techniques for Large Linear Systems: A Survey, Journal of Computation Physics, 182, pp. 418-477.

Borges, C. L., \& Coutinho, A. L. (1996). Solução de Fluxo de Potência em Ambiente Vetorial usando usando o Método do Gradiente Bi-Conjugado Estabilizado.In: 11o. Congresso Brasileiro de Automática, São Paulo, Anais do 11o. Congresso Brasileiro de Automática, v. 1. p. $160-166.11$ o.

Bruaset, A. M. (1995). A Survey of Preconditioned Iterative Methods. Harlow, UK: Longman Scientific \& Technical.

CEPEL. (2001). Curso de Treinamento - Programa ANATEM.

Chen, K. (2005). Matrix Preconditioning Techniques and Applications. Cambridge Monographs on Applied and Computational Mathematics.

Cuthill, E. (1972). Several strategies for reducing the bandwidth of matrices. D. J. Rose and R. A. Willoughby, Plenum, New York.

Flueck, A.J. \& Chiang, H.D. (1998). Solving the nonlinear power flow equations with an inexact Newton method using GMRES. IEEE Transactions on Power Systems, vol. 13 , no. 2 , pp. 267-273.

Greenbaum, A. (1997). Iterative Methods for Solving Linear Systems. SIAM, Philadelphia, PA.

Monticelli, A. (1983). Fluxo de Carga em Redes de Energia Elétrica. São Paulo: E Blucher Ltda.
Pessanha, José E. O.; Portugal, Carlos e Saavedra, Osvaldo (2009). Investigação Crítica do Desempenho do GMRES Pré-condicionado via Fatoração Incompleta LU em Estudos de Fluxo de Carga. Sba Controle \& Automação. vol.20, n.4, pp. 564-572.

Prada, R. (1977). Soluções Ajustadas de Fluxo de Potência no Método Rápido Desacoplado, Dissertação de Mestrado, Pontifícia Universidade Católica do Rio de Janeiro.

Saad, Y., \& Schultz, M. H. (1986). GMRES: A generalized minimal residual algorithm for solving nonsymmetric linear systems. pp. SIAM J. Sci. Stat. Comput. Vol. 7, issue 3, pp. 856-869.

Saad, Y. (1994). ILUT: A dual threshold incomplete LU factorization. Numerical Linear Algebra with Applications, Vol. 1 Issue 4, Pages 387 - 402.

Saad, Y. (1996). Iterative Methods for Sparse Linear Systems. Boston: PWS Publishing.

Saad, Y. (2003). Iterative Methods for Sparse Linear System. 2nd edition, SIAM, Society for industrial and applied Mathematics, Philadelphia, PA.

Tinney, W. (1967). Direct solutions of sparse network equations by optimally ordered triangular factorization. Proceedings of the IEEE, Volume: 55, Issue: 11, pp. 1801- 1809.

Van Der Vorst, H. A. (2003). Iterative Krylov methods for large linear systems. Cambridge University Press, Cambridge: vol. 13 of Cambridge Monographs on Applied and Computational Mathematics.

Watkins, D.S. (2002). Fundamentals of Matrix Computations, second edition, Wiley-Interscience Series.

Zhang, J. (2000). A multilevel dual reordering strategy for robust incomplete $\mathrm{LU}$ factorization of indefinite matrices. SIAM Journal on Matrix Analysis and Applications Vol. 22, Issue 3, pp: 925 - 947. 


\section{APÊNDICE A}

Algoritmo básico do Pré-Condicionador ILUT

Passo 1. Para $i=2, \ldots, n$ Fazer:

Passo 2. $\quad w:=a_{i, *}$

Passo 3. Para $\mathrm{k}=1, \ldots, \mathrm{i}-1$ e quando $\mathrm{w}_{k} \neq 0$ Fazer:

Passo 4. $\quad w_{k}:=w_{k} / a_{k k}$

Passo 5. Aplicar a regra para preencher ou descartar $\mathrm{w}_{k}$

Passo 6. $\quad$ Se $\mathrm{w}_{k} \neq 0$ Então:

Passo $7 . \quad$ Para $\mathrm{j}=\mathrm{k}+1, \ldots, \mathrm{n}$ Fazer:

Passo 8. $\quad \mathrm{w}_{j}:=\mathrm{w}_{j}-\mathrm{w}_{k} \mathrm{u}_{k j}$

Passo 9.

Passo 10.

Passo 11.

Passo 12.

Passo 13.

Passo 14.

Passo 15.

Passo 16. Fim - Fazer

\section{APÊNDICE B}

\section{Dados dos Pré-condicionadores}

Tabela B.I: Parâmetros da regra convencional

\begin{tabular}{|c|c|c|}
\hline Parâmetro & Tinney II & RCM \\
\hline \hline$\tau$ & $4,1 \times 10^{-12}$ & $1,0 \times 10^{-7}$ \\
\hline$\rho$ & 95 & 18 \\
\hline
\end{tabular}

Tabela B.II: Parâmetros da regra composta

\begin{tabular}{|c|c|c|}
\hline Parâmetro & Regra Convencional & Regra Baseada no Erro \\
\hline \hline$\tau$ & $4,1 \times 10^{-12}$ & $4,32 \times 10^{-7}$ \\
\hline$\xi$ & - & $1,89 \times 10^{-4}$ \\
\hline$\rho$ & 95 & 92 \\
\hline
\end{tabular}

